\title{
The next generation space VLBI project, VSOP-2
}

\author{
Yasuhiro Murata ${ }^{1}$, Nanako Mochizuki $^{1}$, Hirofumi Saito ${ }^{1}$, \\ Hisashi Hirabayashi ${ }^{1}$, Makoto Inoue ${ }^{2}$, Hideyuki Kobayashi $^{2}$, \\ Philip G. Edwards ${ }^{3}$ and the Next Generation Space \\ VLBI Working Group \\ ${ }^{1}$ Institute of Space and Astronautical Science, Japan Aerospace Exploration Agency, \\ 3-1-1 Yoshinodai, Sagamihara, Kanagawa 229-8510, Japan. \\ email:murata@vsop.isas.jaxa.jp \\ ${ }^{2}$ National Astronomical Observatory of Japan, 2-21-1 Osawa, Mitaka, Tokyo 181-8588, Japan \\ ${ }^{3}$ CSIRO, Australia Telescope National Facility, Locked Bag 194, Narrabri, \\ NSW 2390, Australia
}

\begin{abstract}
The first dedicated space-VLBI project, the VLBI Space Observatory Programme (VSOP), commenced with the successful launch of radio-astronomical satellite HALCA in 1997. Plans for a second generation space-VLBI project have been made by a working group over a number of years. This project, VSOP-2, has now been approved by Japan's space agency, JAXA, as the ASTRO-G project. It is planned for the spacecraft to observe in the 8,22 and $43 \mathrm{GHz}$ bands with cooled receivers for the two higher bands, which include important maser lines. It will have a maximum angular resolution at $43 \mathrm{GHz}(7 \mathrm{~mm})$ of about 40 micro-arcseconds. Although the VSOP project mainly observed continuum emission from active galactic nuclei (AGN), VSOP-2/ASTRO-G is expected to enable a variety of high angular resolution maser line observations.
\end{abstract}

Keywords. space vehicles, instrumentation: high angular resolution, telescopes

\section{VSOP-1/HALCA project}

The Institute of Space and Astronautical Science (ISAS) started the VLBI Space Observatory Programme (VSOP) in 1989, and launched the first space VLBI satellite, HALCA, in 1997. Launch was on the first flight of the M-V rocket (Hirabayashi et al. 1999, Hirabayashi et al. 2000)

HALCA carried an $8 \mathrm{~m}$ diameter center-fed Cassegrain mesh antenna. The on-board radio astronomy subsystem was composed of low-noise amplifiers for three frequency bands, $1.60-1.73 \mathrm{GHz}, 4.7-5.0 \mathrm{GHz}$ and $22.0-22.3 \mathrm{GHz}$. The $22 \mathrm{GHz}$ system temperature was found to be unexpectedly high, and we could get space-ground fringes only for a bursting Orion-KL maser.

Scientific observations were undertaken at 1.6 and $5 \mathrm{GHz}$, which provided the highest angular resolution images ever obtained in these frequency bands $(0.36$ mas at $5 \mathrm{GHz}$, and 1.1 mas at $1.6 \mathrm{GHz}$ ). VSOP observations were mostly devoted to AGN, although several observations were made of non-AGN sources, such as hydroxyl $(\mathrm{OH})$ masers, pulsars, and X-ray binaries. VSOP observations yielded new results on topics such as the internal structure and motion of AGN jets, the highest brightness temperature sources, and the fine structure of the plasma shadow of free-free absorption. 


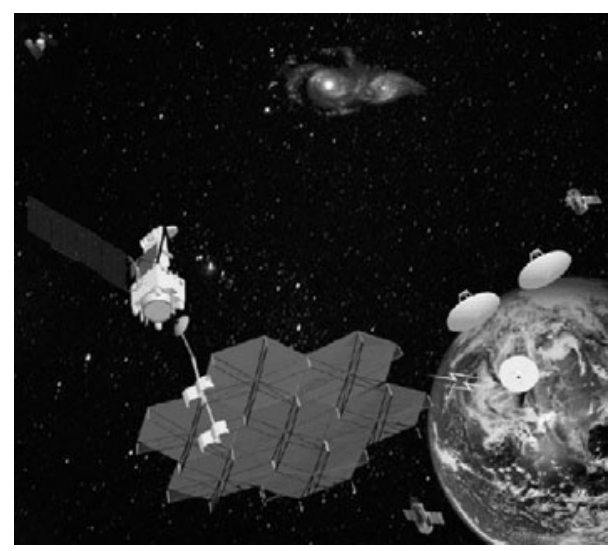

Figure 1. Schematic view of the ASTRO-G (VSOP-2) satellite.

In total, 750 VSOP observations were successfully made. The HALCA satellite lost attitude in October, 2003, and satellite operations were finally ended in November 2005, on the 3213th day after launch.

\section{VSOP-2/ASTRO-G project}

A next-generation space VLBI mission Working Group, which consists of the space VLBI research community, was established by ISAS in May 1997, shortly after fringes were first found to HALCA. The working group submitted a VSOP-2 mission proposal to ISAS in September 2005. The VSOP-2 mission (Hirabayashi et al. 2000) was selected as the 25th scientific mission of JAXA in May 2006 and the VSOP-2 satellite given the developmental name ASTRO-G. VSOP/HALCA was formally an ISAS engineering test mission, but VSOP-2 has been selected as a ISAS/JAXA science mission. To support the ambitious science goals, the ASTRO-G spacecraft requires improvements in both sensitivity and angular resolution when compared with HALCA.

The VSOP-2 spacecraft, ASTRO-G (figure 1), will have a deployable 9-m off-axis paraboloid antenna with an uncooled receiver operating in the range $8.0-8.8 \mathrm{GHz}$, and cryogenically cooled receivers operating from $20.6-22.6 \mathrm{GHz}$ and $41-45 \mathrm{GHz}$ in both LHCP and RHCP.

To achieve an order of magnitude higher sensitivity for continuum sources, VLBI data will be down-linked in real-time at 1 gigabit per second using the $37-38 \mathrm{GHz}$ band. The on-board system is locked to a reference phase, derived from a $\mathrm{H}$-maser at one of $3 \sim 4$ tracking stations, and uplinked as a tone at $40 \mathrm{GHz}$. ASTRO-G has 2 IF channels with 2 sampling modes. One uses $256 \mathrm{MHz}$ bandwidth, 1-bit sampled channels, and the other has $128 \mathrm{MHz}$, 2- bit sampled channels.

The satellite will be placed in an elliptical orbit with an apogee height of $25,000 \mathrm{~km}$ above the Earth's surface and a perigee height of $1,000 \mathrm{~km}$, resulting in a period of 7.5 hours.

With an apogee height of $25,000 \mathrm{~km}, 43 \mathrm{GHz}$ observations can achieve an angular resolution of 38 micro-arcseconds. Furthermore, a phase-referencing capability is being actively considered which will not only increase the number of observable sources but will also allow state-of-the-art astrometric measurements to be undertaken. ASTRO-G should achieve an order of magnitude higher sensitivity than HALCA with these new capabilities. Table 1 shows the target sensitivities for line and continuum sources. 
Table 1. Target VSOP-2 observational parameters

\begin{tabular}{|c|c|c|c|}
\hline Observing Frequency & $8 \mathrm{GHz}$ & $22 \mathrm{GHz}$ & $43 \mathrm{GHz}$ \\
\hline Resolution & 205 mas & 75 mas & $38 \mathrm{mas}$ \\
\hline Target SEFD & $4080 \mathrm{Jy}$ & $2200 \mathrm{Jy}$ & $3170 \mathrm{Jy}$ \\
\hline Target Tsys & $60 \mathrm{~K}$ & $30 \mathrm{~K}$ & $40 \mathrm{~K}$ \\
\hline \multicolumn{4}{|l|}{ Line sensitivity } \\
\hline Target Line & - & $\mathrm{H}_{2} \mathrm{O}$ & $\mathrm{SiO}$ \\
\hline \multicolumn{4}{|c|}{$7 \sigma$ Fringe detection sensitivity $(1 \mathrm{~km} / \mathrm{s}$ line width $)$} \\
\hline $\begin{array}{l}\text { with VLBA } 25 \mathrm{~m} \\
\text { with phased-VLA }\end{array}$ & - & $\begin{array}{r}2.9 \mathrm{Jy} \\
0.66 \mathrm{Jy}\end{array}$ & $\begin{array}{r}4.5 \mathrm{Jy} \\
0.93 \mathrm{Jy}\end{array}$ \\
\hline \multicolumn{4}{|l|}{ Phase referencing sensitivity } \\
\hline 90 min., VLBA $25 \mathrm{~m}$ & - & $0.43 \mathrm{Jy}$ & $0.47 \mathrm{Jy}$ \\
\hline \multicolumn{4}{|l|}{ Continuum sensitivities } \\
\hline \multicolumn{4}{|l|}{$7 \sigma$ Fringe detection sensitivity } \\
\hline with VLBA $25 \mathrm{~m}$ & $25 \mathrm{mJy}$ & $20 \mathrm{mJy}$ & $110 \mathrm{mJy}$ \\
\hline with phased-VLA & 5 mJy & 11 mJy & $22 \mathrm{mJy}$ \\
\hline \multicolumn{4}{|l|}{ Phase referencing sensitivity } \\
\hline 90 min., VLBA $25 \mathrm{~m}$ & $6 \mathrm{mJy}$ & $8 \mathrm{mJy}$ & 11 mJy Jy \\
\hline Image sensitivity (12hour) & $0.034 \mathrm{mJy} / \mathrm{beam}$ & $0.064 \mathrm{mJy} / \mathrm{beam}$ & $0.100 \mathrm{mJy} / \mathrm{beam}$ \\
\hline Detectable Brightness temperature & $6.8 \times 10^{7} \mathrm{~K}$ & $1.3 \times 10^{8} \mathrm{~K}$ & $2.1 \times 10^{8} \mathrm{~K}$ \\
\hline
\end{tabular}

The design of the VSOP-2 instruments is intended to realize the following science goals: (i) The structures and magnetic field configurations of accretion disks in nearby active galactic nuclei (AGNs), (ii) The mechanism of jet acceleration and collimation, (iii) The motion of masers in galactic star forming regions, (iv) The study of proto-stellar magnetospheres, and (v) The structures and magnetic fields of accretion disks in active galactic nuclei.

As a result, VSOP-2 will allow studies of regions where extreme physical conditions are encountered. Consequently the high-resolution imaging capability of VSOP-2 will enable new science in fundamental astrophysics to be undertaken.

\section{VSOP-2 maser observations}

Extragalactic water masers are found within a few parsecs of several active galactic nuclei. $\mathrm{H}_{2} \mathrm{O}$ masers in the spiral galaxy NGC 4258 are located 40,000 Schwarzschild radii from the central engine in a rapidly rotating accretion disk. Monitoring observations to determine the three dimensional motions of these maser spots were used to prove the existence of a supermassive black hole in the center of the galaxy. The motions of such spots allows not only the supermassive black hole mass in the AGN center to be obtained but also the temperature and density of the emitting gas to be probed, revealing the physical conditions of the accretion disk. At present about 50 extragalactic water 'megamaser' sources are known. An advantage of VSOP-2 observations for megamasers is, again, the ability to measure the proper motions of the maser spots in a shorter time than required for ground-based observations.

$\mathrm{H}_{2} \mathrm{O}$ and $\mathrm{SiO}$ masers observations are a main target of the VSOP-2 project. Candidate objects to observe with those maser lines are galaxies, low and high mass star forming regions, and late-type stars (Mira type stars, $\mathrm{OH} / \mathrm{IR}$ stars and planetary nebulae) in our Galaxy. Masers in the nuclei of low redshift $(z<0.05)$ galaxies, and emissions from Local group galaxies, such as the LMC, SMC, and M33, can also be studied. 


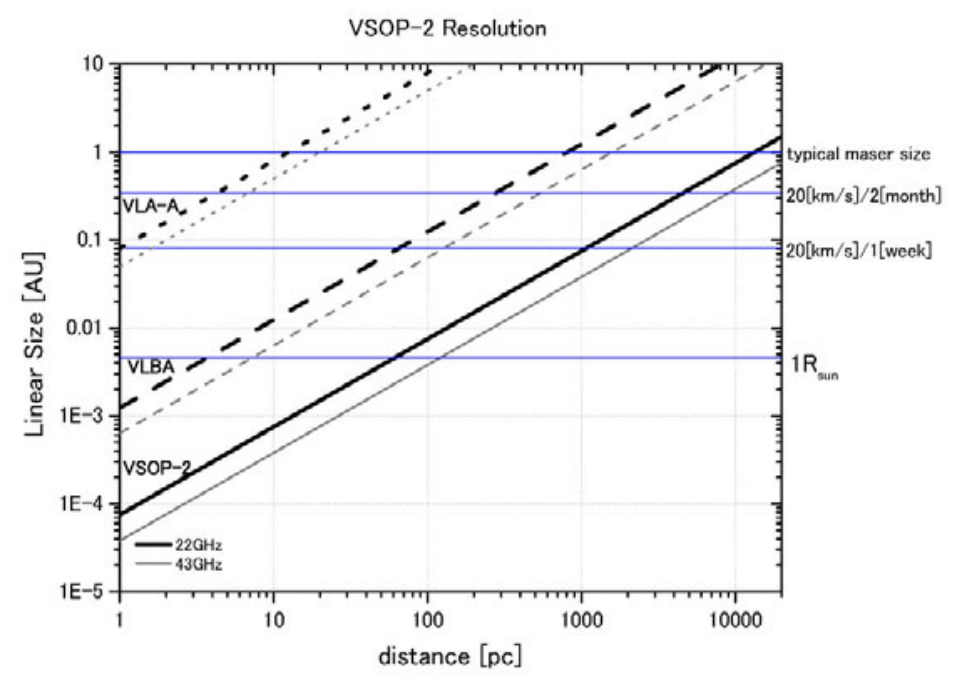

Figure 2. Diagram indicating how the VSOP-2 angular resolution compares with sources in our Galaxy. The horizontal axis shows the distance from the sun, and the vertical axis shows the absolute size of the sources.

By combining phase referencing VLBI or Space-VLBI observations with radial velocity information derived from the maser spot Doppler shift, it is possible to reconstruct the the 3-D motions of the maser spots. Such 3-D motions are required for determining the gravitational field in which gas is moving and for the study of other environmental conditions.

In star forming regions, water maser motions appear as an outflow from the protostellar disk which allows important constraints to be placed on star formation mechanisms. Compared to ground-VLBI observations, VSOP-2 observations have the distinct advantage of being able to measure proper motions over a shorter period of time, and, as a result, will enable shorter-lifetime maser spot motions to be determined. We show how small scale we can measure with VSOP-2 on figure 2.

$\mathrm{H}_{2} \mathrm{O}$ and $\mathrm{SiO}$ masers associated with late type stars probe an important stage in stellar evolution. $\mathrm{H}_{2} \mathrm{O}$ and several $\mathrm{SiO}$ maser transitions fall in the observing band of VSOP-2. Multi-transitional observations of these $\mathrm{SiO}$ masers excited in different inversion schemes will give new information on the local physical conditions of the maser emission, thereby tracing the mass loss process.

One of the problems when we observe Galactic maser emission with VSOP-2 is its spot size. Larger size spots cannot be detected on the longest baselines as the sources are resolved out. From figure 3 of Gwinn et al. (1988), the size of nearby/neighborhood sources are large, but more distant sources are smaller with the exception of sources near the Galactic center direction because of the scatter broadening. The VSOP pre-launch maser survey of Migenes et al. (1999) pointed out there is a large range of angular sizes of maser spots from 10 mas to less than 0.1 mas. Though it is difficult to observe larger size spots by space VLBI, there are still many targets with smaller size spots. We think new pre-launch survey of maser spots will be useful for VSOP-2 maser observations.

\section{Current status of VSOP-2/ASTRO-G}

VSOP-2 received a new start at the beginning of Japanese 2007 fiscal year and funding is currently in place for satellite development. A schedule to develop ASTRO-G is shown 


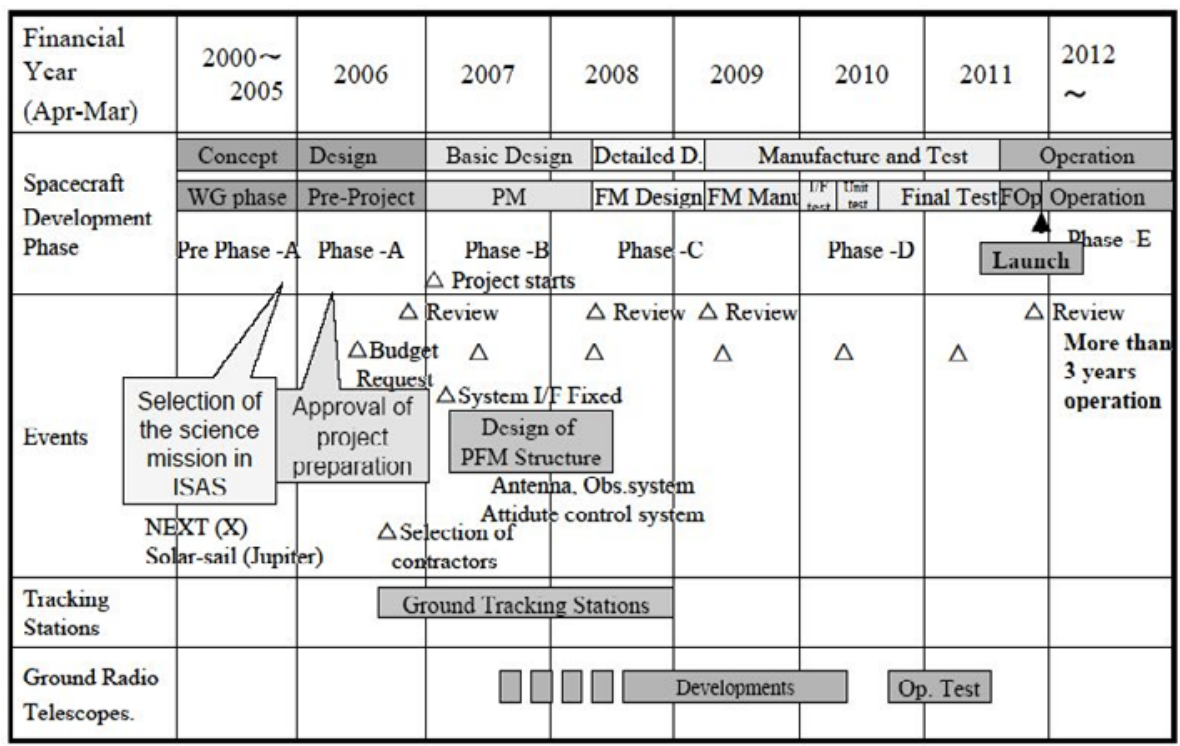

Figure 3. Current development schedule of ASTRO-G/VSOP-2 satellite.

in Figure 3. The current launch date is early 2012. A mission lifetime of more than 3 years is planned, and hopefully more than the 6 years of HALCA. We have now almost finished its concept design, and will move to the more detailed design of the satellite, such as the location of the instruments, and defining the mechanical and electrical interfaces. We also start to configure the international collaboration in basically the same manner as for VSOP-1.

\section{Summary}

The budget for the VSOP-2 (ASTRO-G) project has been approved with the target launch date of 2012. Reviews to approve the start of the Phase B stage (engineeringmodel development) were held in March/April 2007. Extragalactic masers and compact Galactic maser sources are important targets of VSOP-2.

\section{Acknowledgements}

We would like to acknowledge all those who are working on, or have contributed to, the VSOP-2 project.

\section{References}

Gwinn, C. R., Moran, J. M., Reid, M. J., \& Schneps, M. H. 1988, ApJ 330, 817

Migenes, V., Horiuchi, S., Slysh, V. I., Val'tts, I. E., Golubev, V.V, Edwards, P. G., Fomalont, E. B., Okayasu, R., Diamond, P. J., Umemoto, T., Shibata K. M., \& Inoue, M. 1999, ApJS 123,487

Hirabayashi et al. 1998, Science 281, 1825 (erratum 282, p. 1995, 1998)

Hirabayashi et al. 2000, PASJ 52, 955

Hirabayashi, H. Murata, Y., Asaki, Y., Edwards, P. G., Mochizuki, N., Natori, M. C., Inoue, M., Umemoto, T., Kameno, S. Kono, Y., Tsuboi, M., Kasuga, T., 2004, Optical, Infrared, and Millimeter Space Telescopes. Edited by Mather, John C. Proceedings of the SPIE 5487, 1646. 